\title{
AlBERT FATHI
}

\section{Démonstration d'un théorème de Penner sur la composition des twists de Dehn}

Bulletin de la S. M. F., tome 120, no 4 (1992), p. 467-484

$<$ http://www.numdam.org/item?id=BSMF_1992_120_4_467_0>

(C) Bulletin de la S. M. F., 1992, tous droits réservés.

L'accès aux archives de la revue «Bulletin de la S. M. F. » (http: //smf.emath.fr/Publications/Bulletin/Presentation.html) implique l'accord avec les conditions générales d'utilisation (http://www.numdam.org/ conditions). Toute utilisation commerciale ou impression systématique est constitutive d'une infraction pénale. Toute copie ou impression de ce fichier doit contenir la présente mention de copyright.

\section{NumDam}




\title{
DÉMONSTRATION D'UN THÉORÈME DE PENNER SUR LA COMPOSITION DES TWISTS DE DEHN
}

\author{
PAR
}

\author{
Albert FATHI (*)
}

\begin{abstract}
RÉSUMÉ. - Nous démontrons le théorème suivant de Penner : si $\left(\gamma_{1}, \ldots, \gamma_{k}\right)$ et $\left(\delta_{1}, \ldots, \delta_{\ell}\right)$ sont deux multicourbes qui remplissent une surface orientée, tout composé en les twists de Dehn positifs $D_{\gamma_{1}}, \ldots, D_{\gamma_{k}}$ et négatifs $D_{\delta_{1}}^{-1}, \ldots, D_{\delta_{\ell}}^{-1}$ autour des courbes $\gamma_{1}, \ldots, \gamma_{k}, \delta_{1}, \ldots, \delta_{\ell}$, qui contient au moins une occurence de chacun d'entre eux, est pseudo-Anosov. La démonstration que nous en donnons ne repose pas sur une théorie du réseau ferroviaire dual. De plus le représentant, dans la classe d'isotopie, que nous construisons est conjugué au pseudo-Anosov par une semi-conjugaison qui n'a qu'un nombre fini de préimages non-réduites à un point.

ABstract. - We prove the following theorem due to Penner : if $\left(\gamma_{1}, \ldots, \gamma_{k}\right)$ and $\left(\delta_{1}, \ldots, \delta_{1}\right)$ are two multicurves filling an oriented surface, every composition of positive Dehn twists $D_{\gamma_{1}}, \ldots, D_{\gamma_{k}}$ and of negative Dehn twists $D_{\delta_{1}}^{-1}, \ldots, D_{\delta_{t}}^{-1}$ around the curves $\gamma_{1}, \ldots, \gamma_{k}, \delta_{1}, \ldots, \delta_{\ell}$, which contains at least one occurence of each of them, is pseudo-Anosov. Our proof does not rely on any theory for the dual train-track. Moreover, we construct a representative in the isotopy class that is semi-conjugate to the pseudo-Anosov representative by a semi-conjugacy with only a finite number of preimages which are not points.
\end{abstract}

\section{Introduction et rappels}

Pour la suite, nous considérons une surface $M$ compacte, connexe, de genre $\geq 2$ et orientée. Nous renvoyons à $[F L P]$ pour la plupart des définitions. Nous faisons les rappels suivants essentiellement pour fixer les notations.

(*) Texte reçu le 10 janvier 1991, révisé le 6 janvier 1992.

A. FAthi, University of Florida, Dept. of Mathematics, 201 Walker Hall, Gainesville, Florida 32611 USA et Laboratoire de Mathématique, ENS Lyon, 46 , Allée d'Italie, 69364 Lyon Cedex 07 France.

Recherche partiellement financée par les bourses \# DMS-8801749 et \# DMS-9101911 de la NSF.

Classification AMS : 57 M 99, 57 N 05, 57 R 50.

Keywords : pseudo-Anosov, twist de Dehn, surface.

BUlletin DE LA SOCiÉtÉ MATHÉMATIQUE DE FRANCE 0037-9484/1992/467/\$5.00

(C) Société mathématique de France 
On note par $\mathcal{S}(M)$ l'ensemble des classes d'isotopie de courbes simples de $M$ non homotopes à 0 ou à une composante du bord. Puisque $M$ est orientée, pour $\gamma$ dans $\mathcal{S}(M)$, on peut définir le twist de Dehn positif $D_{\gamma}$ autour de $\gamma$ comme classe d'isotopie de difféomorphismes. Le twist négatif autour de $\gamma$ est bien sûr l'inverse du twist positif. On note $\mathcal{S}^{\prime}(M)$ l'ensemble des classes d'isotopie de sous-variétés compactes de dimension 1 de $M$ dont aucune composante connexe n'est homotope à 0 ou à une composante du bord. Un élément $\Gamma \in \mathcal{S}^{\prime}(M)$ peut donc être identifié à une suite $\left(\gamma_{1}, \ldots, \gamma_{k}\right)$ où $\gamma_{1}, \ldots, \gamma_{k} \in \mathcal{S}(M)$ et $i\left(\gamma_{h}, \gamma_{\ell}\right)=0,1 \leq h, \ell \leq k$. Une famille $\left\{\eta_{1}, \ldots, \eta_{n}\right\}$ d'éléments de $\mathcal{S}(M)$ remplit la surface $M$, si pour toute classe $\gamma \in \mathcal{S}(M)$ il existe un $\eta_{i}$ tel que $i\left(\gamma, \eta_{i}\right) \neq 0$; de manière équivalente $\left[\mathrm{Fa}\right.$, Th. 3.4, p. 133] la famille $\left\{\eta_{1}, \ldots, \eta_{n}\right\}$ remplit la surface si, pour tous représentants $e_{1}, \ldots, e_{n}$ de $\eta_{1}, \ldots, \eta_{n}$, les composantes de $M \backslash \bigcup_{i=1}^{i=n} e_{i}$ sont, soit des disques ouverts, soit des anneaux (semiouvert) dont le bord est une composante du bord de $M$.

Le théorème suivant est dû à PENner $[\mathrm{Pe}]$ :

ThÉorème. - Soient $\Gamma=\left(\gamma_{1}, \ldots, \gamma_{k}\right)$ et $\Delta=\left(\delta_{1}, \ldots, \delta_{l}\right)$ dans $\mathcal{S}^{\prime}(M)$, où $M$ est une surface compacte connexe et orientée. Supposons que $\left\{\gamma_{1}, \ldots, \gamma_{k}, \delta_{1}, \ldots, \delta_{\ell}\right\}$ remplissent $M$. Alors, tout composé en les twists de Dehn positifs autour des courbes dans $\Gamma$ et en les twists de Dehn négatifs autour des courbes dans $\Delta$, qui contient au moins une occurence de chaque courbe dans $\left\{\gamma_{1}, \ldots, \gamma_{k}, \delta_{1}, \ldots, \delta_{\ell}\right\}$, définit une classe d'homotopie de difféomorphismes qui contient un difféomorphisme pseudo-Anosov.

Nous nous proposons de donner ici une démonstration qui n'utilise pas la théorie des réseaux ferroviaires. En particulier, nous ne faisons pas appel à une théorie du réseau ferroviaire dual.

Notre démonstration est, en esprit, plus proche de la démonstration de Thurston du fait que le composé de twists autour de deux courbes, qui remplissent, est pseudo-Anosov. Nous utilisons la structure plate définie par $\Gamma$ et $\Delta$ pour choisir des représentants affines par morceaux des twists. Une analyse directe montre les propriétés d'allongement des courbes. Le théorème de classification de Thurston permet de conclure. Dans la suite, nous donnons une représentation matricielle qui permet de calculer les coefficients de dilatation. Nous montrons aussi que les représentants affines par morceaux dans la structure plate sont pratiquement pseudo-Anosov.

Ce qui suit peut aussi se faire pour une surface à bord. Pour plus de clarté, nous supposerons que $\partial M=\emptyset$.

Ce travail n'aurait jamais vu le jour sans l'intêret que lui a témoigné François LAUdenbach. Je le remercie pour cela aussi.

TOME $120-1992-\mathrm{N}^{\circ} 4$ 


\section{La structure plate associée à deux multicourbes}

Soient $\Gamma=\left(\gamma_{1}, \ldots, \gamma_{k}\right)$ et $\Delta=\left(\delta_{1}, \ldots, \delta_{\ell}\right)$ deux multicourbes qui remplissent $M$. Choisissons des représentants $c_{j} \in \gamma_{j}, j=1, \ldots, k$ et $d_{h} \in \delta_{h}, h=1, \ldots, \ell$ qui minimisent les intersections géométriques, i.e.

$$
\#\left(c_{i} \cap d_{j}\right)=i\left(\gamma_{i}, \delta_{j}\right), \quad \#\left(c_{i} \cap c_{h}\right)=i\left(\gamma_{i}, \gamma_{h}\right)=0
$$

et de même \# $\left(d_{j} \cap d_{k}\right)=i\left(\delta_{j}, \delta_{k}\right)=0$.

Comme dans [FLP, exposé 13, III.1, p. 245], on définit une structure plate sur $M$ pour laquelle les feuilletages mesurés associés à $\Gamma$ et $\Delta$ apparaissent, respectivement, comme le feuilletage mesuré horizontal $\mathcal{H}$ et le feuilletage mesuré vertical $\mathcal{V}$. De plus, les $c_{j}$ sont des feuilles lisses de $\mathcal{H}$ et les $d_{h}$ des feuilles lisses de $\mathcal{V}$.

Cette structure plate est obtenue en recollant des carrés de côté de longueur 1, ayant chacun deux côtés horizontaux et deux côtés verticaux. Le feuilletage $\mathcal{H}$ (resp. $\mathcal{V}$ ) représente donc $\Gamma$ (resp. $\Delta$ ) dans $\mathcal{M F}(M)$.

Cette structure plate donne une métrique et une mesure des angles en chaque point, y compris les points singuliers. En un point singulier $p$, l'angle total autour de $p$ est $n \pi$ ou $n$ est le nombre de séparatrices $\mathrm{du}$ feuilletage horizontal (ou vertical). Remarquons que les feuilletages horizontaux et verticaux sont partout orthogonaux.

Les géodésiques de la métrique plate sont constituées de segments rectilignes (lisses) qui se joignent en des singularités. En une telle singularité, les deux angles découpés par la géodésique sont $\geq \pi$. Toute classe d'homotopie de chemins à extrémités fixées contient une unique géodésique de cette structure plate. On peut consulter [St] au sujet des géodésiques de ces structures plates; remarquons, toutefois, que nous n'utilisons pas la même convention pour mesurer les angles aux points singuliers.

Si $m \in M$ est un point régulier de la structure plate, on peut trouver une carte locale $(U, x, y)$, où $U$ est un voisinage de $m$ et $x, y: U \rightarrow \mathbb{R}$ sont deux fonctions numériques, telle que $n \mapsto(x(n), y(n))$ soit un difféomorphisme préservant l'orientation de $U$ sur un voisinage de 0 dans $\mathbb{R}^{2}$ qui envoie $m$ sur 0 et tel que le feuilletage mesuré horizontal (resp. vertical) y soit donné par la 1 -forme fermée $\mathrm{d} y$ (resp. $\mathrm{d} x)$. Si $(V, X, Y)$ est une autre telle carte, on a $X=-x$ et $Y=-y$ dans $U \cap V$. Il en résulte que le cône positif

$$
C_{+}(m)=\left\{v \in T_{m} M \mid \mathrm{d} x(v) \mathrm{d} y(v) \geq 0\right\}
$$

(resp. le cône négatif $C_{-}(m)=\left\{v \in T_{m} M \mid \mathrm{d} x(v) \mathrm{d} x(v) \leq 0\right\}$ ) est bien défini au point régulier $m$ de la structure plate. Chacun de ces cônes est composé de deux secteurs (voir figure 1). En un point singulier, de la structure plate, on peut aussi définir les "cônes positifs" et négatifs de 
manière analogue. En une telle singularité, un cône a $n$ secteurs, où $n$ est le nombre de séparatrices du feuilletage horizontal (ou vertical). Autour d'un point, les secteurs positifs et négatifs alternent.

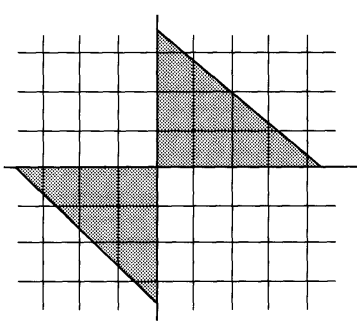

cône positif

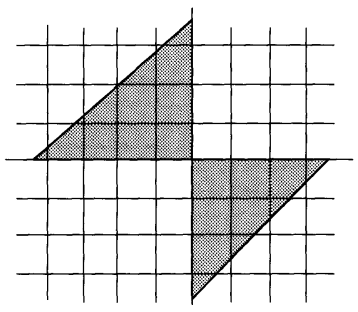

cône négatif

Figure 1.

Pour chaque $j=1, \ldots, k$, il est possible de représenter la classe d'isotopie du twist de Dehn positif autour de $\gamma_{j}$ par un homéomorphisme $T_{j}$, qui est affine sur l'anneau de feuilles horizontales parallèles à $c_{j}$ et est l'identité ailleurs (figure 2). De la même façon, pour chaque $h=1, \ldots, \ell$, il est possible de représenter la classe d'isotopie du twist de Dehn positif autour $\delta_{h}$ par un homéomorphisme $S_{h}$, qui est affine sur l'anneau de feuilles parallèles à $d_{h}$ et est l'identité ailleurs.

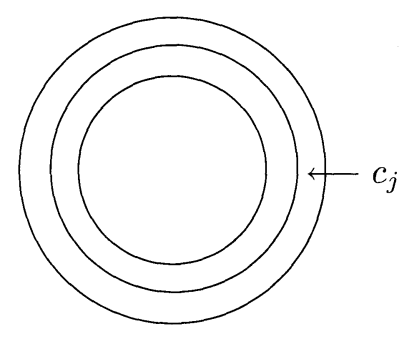

Figure 2. Le support de $T_{j}$ est l'anneau de feuilles homotopes à $c_{j}$.

Le lemme suivant est évident :

LEMME 1.1. - Les homéomorphismes $T_{j}$ et $S_{h}$ sont affines par morceaux. De plus, l'image par $T_{j}$ (resp. $S_{h}^{-1}$ ) d'un secteur du cône positif au point $m$ de la structure plate est contenue dans un secteur du cône positif au point $T_{j}(m)$ (resp. $\left.S_{h}^{-1}(m)\right)$. De même, l'image par $T_{j}^{-1}\left(\right.$ resp. $\left.S_{h}\right)$ d'un secteur du cône négatif en $m$ est contenue dans un secteur du cône négatif au point $T_{j}^{-1}(m)$ (resp. $\left.S_{h}(m)\right)$. 


\section{Chemins positifs ou négatifs}

Rappelons qu'un chemin $c:[0,1] \rightarrow M$ (où $c(0)=c(1)$ ) est $\mathrm{C}^{1}$ par morceaux, s'il existe une suite $a_{0}=0, a_{1}, \ldots, a_{n}=1$ telle que $c$ soit $\mathrm{C}^{1}$ sur chaque intervalle fermé $\left[a_{i}, a_{i+1}\right], i=0, \ldots, n-1$. Pour un tel chemin, le vecteur tangent à droite et le vecteur tangent à gauche existent en chaque point. Bien sûr, il sont égaux sauf peut-être aux points $c\left(a_{i}\right), i=0, \ldots, n$.

DÉfinition 2.1 (Chemin positif). - Un chemin $c$ est appelé positif si :

(i) c'est un chemin $\mathrm{C}^{1}$ par morceaux;

(ii) en chaque point, le vecteur tangent à droite et l'opposé du vecteur tangent à gauche au chemin sont contenus dans des secteurs différents du cône positif.

Un chemin strictement positif est un chemin positif tel que les vecteurs tangents à droite et à gauche au chemin sont contenus dans l'intérieur du cône positif.

Les chemins négatifs et strictement négatifs sont définis d'une manière analogue.

Remarquons qu'un chemin strictement positif (resp. négatif) est quasitransverse à la fois au feuilletage horizontal et au feuilletage vertical.

En fait, les chemins que l'on utilisera par la suite seront géodésiques par morceaux dans la structure plate.

Lemme 2.2. - Soient $\alpha$ et $\beta$ deux chemins homotopes à extrémités fixées. Supposons que $\alpha$ est strictement positif (resp. négatif), on a:

(i) le chemin $\beta$ n'est pas strictement négatif (resp. positif);

(ii) si le chemin $\beta$ est une géodésique de la structure plate, alors $\beta$ est strictement positif (resp. négatif).

Preuve. - On peut supposer que $\alpha$ et $\beta$ sont distincts.

Démontrons (i) par l'absurde : supposons que $\beta$ soit strictement négatif. Dans le revêtement universel de $M$, nous pouvons trouver un disque $D$ dont le bord est réunion de deux segments $\alpha_{1}$ et $\beta_{1}$, respectivement, contenus dans des relevés de $\alpha$ et $\beta$ au revêtement universel. Remarquons que, si nous regardons un point d'intersection de $\alpha_{1}$ et $\beta_{1}$, nous voyons qu'au moins un des deux feuilletages horizontal ou vertical possède une feuille qui entre dans le disque. Si cette feuille n'aboutit pas à une singularité dans l'intérieur du disque, elle recoupe le bord. On obtiendrait alors un disque avec un bord qui serait réunion d'un morceau de feuille et d'un segment quasi-transverse au feuilletage, mais ceci est impossible, [FLP, p. 77]. Si la feuille aboutit à une singularité, on peut trouver une feuille proche qui conduirait à la même contradiction que ci-dessus. 
Pour démontrer (ii), commençons par le cas où le chemin $\beta$ contient un morceau $f$ d'une feuille horizontale. Dans le revêtement universel de $M$, nous pouvons trouver un disque $D$ dont le bord est réunion de deux segments $\alpha_{1}$ et $\beta_{1}$, respectivement, contenus dans des relevés de $\alpha$ et $\beta$ au revêtement universel,et où $\beta_{1}$ contient un morceau d'un relevé de $f$. Comme les deux angles d'une géodésique formés en un point singulier sont $\geq \pi$, il n'est pas difficile de trouver dans le revêtement universel un disque $D^{\prime} \subset D$ dont le bord est composé de 2 morceaux, un des deux étant inclus dans $\alpha$ et l'autre contenu dans une réunion de feuilles et de points singuliers du feuilletage horizontal. Mais ceci est impossible, car $\alpha$ est quasi-transverse au feuilletage horizontal. De même, on aboutit à une contradiction dans le cas où la géodésique $\beta$ contient un morceau de feuille du feuilletage vertical.

Pour finir la démonstration, il reste à examiner le cas où $\beta$ contient une singularité $s \notin \alpha$, est strictement positif d'un côté de $s$ et strictement négatif de l'autre côté. Dans le revêtement universel de $M$, nous pouvons trouver un disque $D$ dont le bord est réunion de deux segments $\alpha_{1}$ et $\beta_{1}$, respectivement, contenus dans des relevés de $\alpha$ et $\beta$ au revêtement universel, et où $\beta_{1}$ contient un relevé $\tilde{s}$ de $s$. Puisque les deux angles formés par $\beta$ en $s$ sont $\geq \pi$ le disque $D$ contient une paire de secteurs adjacents en $\tilde{s}$, l'un positif et l'autre négatif. Le bord de la réunion de ces deux secteurs consiste en une paire de séparatrices et une singularité d'un des feuilletages horizontal ou vertical. Si on prolonge ces séparatrices en une réunion de feuilles et de singularités, comme plus haut, on trouve une contradiction.

LEMME 2.3. - L'image par $T_{j}$ ou par $S_{h}^{-1}$ d'un chemin positif (resp. strictement positif) est un chemin positif (resp. strictement positif). De même, l'image par $T_{j}^{-1}$ ou par $S_{h}$ d'un chemin négatif (resp. strictement négatif) est un chemin négatif (resp. strictement négatif).

Preuve. - Ceci suit du Lemme 1.1.

Lemme 2.4. - Soient $\alpha$ un chemin contenu dans une feuille du feuilletage horizontal (resp. vertical) sauf éventuellement pour ses extrémités qui peuvent être des singularités. Si $H$ est un composé en les $T_{j}$ et les $S_{h}^{-1}$, alors, soit $H(\alpha)$ est contenu dans la même feuille du feuilletage horizontal (resp. vertical), soit $H(\alpha)$ est homotope à extrémités fixées à un chemin strictement positif.

De même, soit $H^{-1}(\alpha)$ est contenu dans la même feuille du feuilletage horizontal (resp. vertical), soit $H^{-1}(\alpha)$ est homotope à extrémités fixées à un chemin strictement négatif.

Preuve. - Par le Lemme 2.3 et le fait que les $T_{j}$ préservent chaque TOME $120-1992-\mathrm{N}^{\circ} 4$ 
feuille du feuilletage horizontal, il suffit de considérer le cas où $H=S_{h}^{-1}$. Si $S_{h}^{-1}$ n'est pas l'identité sur $\alpha$, le chemin $S_{h}^{-1}(\alpha)$ est composé de segments rectilignes (pour la structure plate) consécutifs $p_{1}, \cdots, p_{n}$ avec $p_{s}$ strictement positif ou $p_{s}$ horizontal, deux segments consécutifs n'étant pas du même type. De plus, si nous nous regardons une paire de segments consécutifs, le segment strictement positif est contenu, près du point d'intersection, dans le secteur du cône positif qui n'est pas adjacent au segment horizontal.
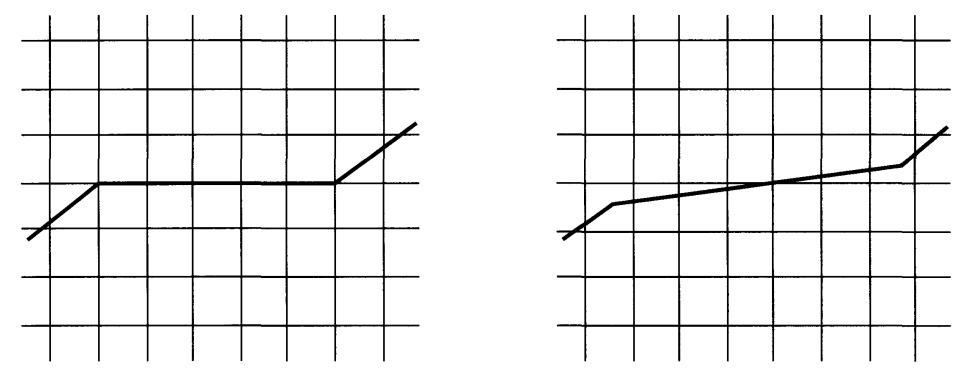

Figure 3.

On se trouve donc dans une situation comme celle illustrée sur le côté gauche de la figure 3 . Sur le côté droit de la figure 3 , nous montrons comment remplacer les segments horizontaux pour obtenir un chemin isotope strictement positif.

Lemme 2.5. - Soit $H$ un composé en les $T_{j}$ et les $S_{h}^{-1}$ et $\alpha$ une géodésique de la structure plate. Si, pour un $n \geq 0$, le chemin $H^{n}(\alpha)$ est homotope à extrémités fixées à un chemin contenu dans une feuille du feuilletage horizontal (resp. vertical), alors soit $H^{n}(\alpha)$ et $\alpha$ sont contenus dans la même feuille du feuilletage horizontal (resp. vertical) soit $\alpha$ est un chemin strictement négatif.

De même, si, pour un $n \geq 0$, le chemin $H^{-n}(\alpha)$ est homotope à extrémités fixées à un chemin contenu dans une feuille du feuilletage horizontal (resp. vertical), alors soit $H^{-n}(\alpha)$ et $\alpha$ sont contenus dans la même feuille du feuilletage horizontal (resp. vertical) soit $\alpha$ est un chemin strictement positif.

Preuve. - Appelons $\beta$ le morceau de feuille homotope à $H^{n}(\alpha)$. Les chemins $\alpha$ et $H^{-n}(\beta)$ sont homotopes à extrémités fixées. Par le LEMme 2.4, soit $H^{-n}(\beta)$ et $\beta$ sont contenus dans la même feuille, soit $H^{-n}(\beta)$ est homotope à extrémités fixées à un chemin strictement négatif. Dans le 
premier cas $\alpha=H^{-n}(\beta)$ par unicité des géodésiques dans une classe d'homotopie à extrémités fixées, et dans ce cas $\alpha=H^{-n}(\beta)$ et $H^{n}(\alpha)=\beta$ sont dans la même feuille. Dans le second cas, le chemin $\alpha$ est homotope à extrémités fixées à un chemin strictement négatif; par le LEMME 2.2, la géodésique $\alpha$ est elle-même strictement négative.

Introduisons les notations suivantes. Si $\alpha$ est un chemin $\mathrm{C}^{1}$ par morceaux, nous notons $\ell_{h}(\alpha)$ (resp. $\ell_{v}(\alpha)$ ) la variation de $\alpha$ pour la mesure transverse associée au feuilletage horizontal (resp. vertical) et $\ell_{s}(\alpha)$ la somme $\ell_{h}(\alpha)+\ell_{v}(\alpha)$.

Il est bien connu [FLP, p. 79] que si $\alpha$ est quasi-transverse aux feuilletages horizontaux et verticaux alors, pour $i=v, h$ ou $s, \ell_{i}(\alpha)$ est le minimum des $\ell_{i}(\beta)$ pour $\beta$ homotope à $\alpha$ avec extrémités fixées. C'est le cas, en particulier, pour $\alpha$ strictement positif ou strictement négatif.

Lemme 2.6. - Si $\alpha$ est un chemin positif (resp. négatif) et $H$ est un composé en les $T_{j}\left(\right.$ resp. $\left.T_{j}^{-1}\right)$ et les $S_{h}^{-1}\left(\right.$ resp. $\left.S_{h}\right)$, on a :

$$
\ell_{h}(H(\alpha)) \geq \ell_{h}(\alpha), \quad \ell_{v}(H(\alpha)) \geq \ell_{v}(\alpha), \quad \ell_{s}(H(\alpha)) \geq \ell_{s}(\alpha) .
$$

De plus, si $\alpha$ est strictement positif (resp. négatif) et n'intersecte en son intérieur aucune séparatrice horizontale ou verticale, on a

$$
\text { soit } H(\alpha)=\alpha, \text { soit } \ell_{s}(H(\alpha)) \geq \ell_{s}(\alpha)+\min \left(\ell_{h}(\alpha), \ell_{v}(\alpha)\right) \text {. }
$$

Preuve. - Il suffit de le démontrer dans le cas où $H$ est un $T_{j}$ ou un $S_{h}$. Auquel cas, c'est évident.

Proposition 2.7. - Soit $H$ un composé en les $T_{j}$ et les $S_{h}^{-1}$ et soit $\gamma$ une classe d'isotopie, non triviale, de courbes fermées simples dans $M$. Appelons a la réunion des segments positifs (resp. négatifs) contenus dans c, la géodésique fermée pour la structure plate dans la classe d'homotopie de $\gamma$. On a :

$$
\forall n \in \mathbb{N}, \quad i\left(H^{n}(\gamma), \mathcal{H}\right) \geq \ell_{h}\left(H^{n}(\alpha)\right), \quad i\left(H^{n}(\gamma), \mathcal{V}\right) \geq \ell_{v}\left(H^{n}(\alpha)\right)
$$

(resp.

$\left.\forall n \in \mathbb{N}, \quad i\left(H^{-n}(\gamma), \mathcal{H}\right) \geq \ell_{h}\left(H^{-n}(\alpha)\right), \quad i\left(H^{-n}(\gamma), \mathcal{V}\right) \geq \ell_{v}\left(H^{-n}(\alpha)\right).\right)$

Preuve. - L'argument est standard. On se place dans le revêtement $N$ de $M$, dont le $\pi_{1}$ est infini cyclique et engendré par $H^{n}(\gamma)$. On relève $H^{n}(c)$ en une courbe fermée $\widehat{H^{n}(c)}$.

TOME $120-1992-\mathrm{N}^{\circ} 4$ 
Remarquons, pour simplifier la suite, que $\widetilde{H^{n}(c)}$ est une courbe simple. En effet, il est bien connu que $c$ se relève en un courbe simple $\tilde{c}$ dans le revêtement $N_{1}$ de $M$, dont le $\pi_{1}$ est infini cyclique et engendré par $\gamma$. De plus, par la théorie des revêtements, on peut relever $H^{n}$ en un homéomorphisme $\widetilde{H^{n}}: N_{1} \rightarrow N$. Or $\widetilde{H^{n}}(\tilde{c})=\widetilde{H^{n}(c)}$.

Le relevé $\widetilde{H^{n}(\alpha)}$ de $H^{n}(\alpha)$, contenu dans $\widetilde{H^{n}(c)}$, est réunion de deux parties $a$ et $b$, où $a$ est composé de segments rectilignes transverses au feuilletage horizontal et $b$ est réunion de segments de feuilles et de singularités du feuilletage horizontal. On a $\ell_{h}\left(H^{n}(\alpha)\right)=\ell_{h}(a)$. Par le Lemme 2.5, une feuille lisse du feuilletage horizontal, qui coupe $a$ en son intérieur, ne peut pas recouper la courbe $\widehat{H^{n}(c)}$. Il en résulte que la réunion $R$ des relevés des feuilles du feuilletage horizontal qui passe par $a$ est une réunion de bandes infinies, deux à deux disjointes, qui joint les deux bouts du cylindre $N$. La somme des longueurs transverses pour le feuilletage horizontal des bandes contenues dans $R$ est évidemment $\ell_{h}(a)$. Toute courbe fermée $d$ non homotope à 0 dans $N$ doit traverser au moins une fois chaque bande dans $R$, puisqu'une telle bande joint les deux bouts du cylindre $N$. Il en résulte que $\ell_{h}(d) \geq \ell_{h}(a)$ pour toute courbe fermée non homotope à 0 dans $N$. Ceci finit la démonstration puisque $i\left(H^{n}(\gamma), \mathcal{H}\right)$ est le minimum des $\ell_{h}(d)$ pour $d$ courbe fermée dans $N$ dans la classe d'homotopie de $\widetilde{H^{n}(c)}$.

\section{Bon mot et expansivité}

DÉfinition 3.1. (Bon mot). - Un bon mot est une composition en les $T_{j}$ et en les $S_{h}^{-1}$ qui contient au moins une occurence de chaque $T_{j}$ et une occurence de chaque $S_{h}^{-1}$.

Lemme 3.2. - Si $H$ est un bon mot et $\alpha$ est un chemin strictement positif, alors, pour tout $n \geq 0$, on a $\ell_{s}\left(H^{n+1}(\alpha)\right)>\ell_{s}\left(H^{n}(\alpha)\right)$.

Si $\beta$ est une feuille fermée lisse ou une connection entre deux singularités du feuilletage vertical, alors $H(\beta)$ est un chemin positif qui contient un sous chemin strictement positif.

Preuve. - Écrivons $H=G_{s} G_{s-1} \cdots G_{1}$, où chaque $G_{i}$ est de la forme $T_{j}$ ou $S_{h}^{-1}$. Puisque $H^{n}(\alpha)$ est aussi strictement positif, il suffit de démontrer la première partie dans le cas $n=0$. Soit $s_{0}$ le premier entier $s$ tel que l'anneau ouvert où $G_{s}$ n'est pas l'identité intersecte $\alpha$. Un tel $s_{0}$ doit exister puisque les $G_{s}$ contiennent au moins une occurence de chaque $T_{j}$ et de chaque $S_{h}^{-1}$. On a $G_{s_{0}-1} \cdots G_{1} \alpha=\alpha$. Le Lemme 2.6 appliqué à l'intersection de $\alpha$ avec l'anneau où $G_{s_{0}}$ n'est pas l'identité donne $\ell_{s}(H(\alpha)) \geq \ell_{s}\left(G_{s_{0}}(\alpha)\right)>\ell_{s}(\alpha)$. 
La démonstration de la seconde partie est presque la même.

Lemme 3.3. - Si $H$ est un bon mot et $\alpha$ est un chemin strictement positif, alors pour tout $n$ assez grand $H^{n}(\alpha)$ intersecte la réunion des séparatrices horizontales ou verticales.

Preuve. - Si c'est faux, alors pour une infinité de $n$, on obtient par 2.6,

- soit $H^{n+1}(\alpha)=H^{n}(\alpha)$;

- soit $\ell_{s}\left(H^{n+1}(\alpha)\right)=\ell_{s}\left(H^{n}(\alpha)\right)+\min \left[\ell_{h}\left(H^{n}(\alpha)\right), \ell_{v}\left(H^{n}(\alpha)\right)\right]$.

Par 3.2, la première alternative n'a pas lieu. Par 2.6, on a

$$
\min \left[\ell_{h}\left(H^{n}(\alpha)\right), \ell_{v}\left(H^{n}(\alpha)\right)\right] \geq \min \left[\ell_{h}(\alpha), \ell_{v}(\alpha)\right]>0,
$$

d'où $\lim _{n \rightarrow+\infty} \ell_{s}\left(H^{n}(\alpha)\right)=+\infty$. Mais, le chemin strictement positif $H^{n}(\alpha)$ étant transverse aux feuilletages horizontal et vertical, dès que $\ell_{h}\left(H^{n}(\alpha)\right)$ ou $\ell_{v}\left(H^{n}(\alpha)\right)$ est 1 , il doit couper au moins deux séparatrices.

Proposition 3.4. - Si $H$ est un bon mot et $\alpha$ est un chemin strictement positif (resp. strictement négatif), alors

$$
\left.\lim _{n \rightarrow+\infty} \ell_{s}\left(H^{n}(\alpha)\right)=+\infty \quad \text { (resp. } \lim _{n \rightarrow+\infty} \ell_{s}\left(H^{-n}(\alpha)\right)=+\infty\right) .
$$

Preuve. - Soit $K$ un entier positif. Il suffit de montrer que pour $n$ assez grand $\ell_{s}\left(H^{n}(\alpha)\right) \geq K$. Découpons $\alpha$ en $2 K+2$ intervalles deux à deux disjoints. Par le lemme précédent, pour tout $n$ assez grand, chacun de ces intervalles intersecte soit une séparatrice verticale soit une séparatrice horizontale. On en conclut que pour $n$ fixé assez grand soit le chemin $H^{n}(\alpha)$ intersecte $(K+1)$ fois la réunion des séparatrices verticales, soit il intersecte $(K+1)$ fois la réunion des séparatrices horizontales. Puisque $H^{n}(\alpha)$ est quasi-transverse aux deux feuilletages, on a soit $\ell_{v}\left(H^{n}(\alpha)\right) \geq K$, soit $\ell_{h}\left(H^{n}(\alpha)\right) \geq K$ (si le chemin $H^{n}(\alpha)$ entre par un bord d'un anneau de feuilles compactes du feuilletage horizontal ou vertical, il doit sortir par l'autre bord).

\section{Démonstration du théorème}

Montrons que, pour tout bon mot $H$ et toute classe $\gamma$ d'homotopie de courbes simples fermées de $M$, on a :

$$
\begin{aligned}
\lim _{n \rightarrow \infty}\left[i\left(H^{n}(\gamma), \mathcal{H}\right)+i\left(H^{n}(\gamma), \mathcal{V}\right)\right]=\infty \\
\text { ou } \lim _{n \rightarrow \infty}\left[i\left(H^{-n}(\gamma), \mathcal{H}\right)+i\left(H^{-n}(\gamma), \mathcal{V}\right)\right]=\infty
\end{aligned}
$$

TOME $120-1992-\mathrm{N}^{\circ} 4$ 
Considérons $c$ la géodésique de la structure plate dans la classe $\gamma$.

Supposons d'abord que la géodésique $c$ contient un segment rectiligne positif. Appelons a la réunion des segments rectilignes positifs inclus dans $c$. Par 2.7, pour tout $n \geq 0$, on a

$$
i\left(H^{n}(\gamma), \mathcal{H}\right)+i\left(H^{n}(\gamma), \mathcal{V}\right) \geq \ell_{h}\left(H^{n}(a)\right)+\ell_{v}\left(H^{n}(a)\right) .
$$

Puisque $H$ est un bon mot, même si $a$ ne contient pas de segment strictement positif, d'après 3.2 , l'image $H(a)$ contient forcément un segment strictement positif. Par 3.4, on obtient

$$
\lim _{n \rightarrow \infty}\left[\ell_{h}\left(H^{n}(a)\right)+\ell_{v}\left(H^{n}(a)\right)\right]=\infty
$$

d'où

$$
\lim _{n \rightarrow \infty}\left[i\left(H^{n}(\gamma), \mathcal{H}\right)+i\left(H^{n}(\gamma), \mathcal{V}\right)\right]=\infty
$$

Dans le cas où la géodésique $c$ ne contient que des segments négatifs, on obtient

$$
\lim _{n \rightarrow \infty}\left[i\left(H^{-n}(\gamma), \mathcal{H}\right)+i\left(H^{-n}(\gamma), \mathcal{V}\right)\right]=\infty
$$

On peut alors appliquer le théorème de classification de Thurston, comme dans [Fa], pour conclure que la classe d'isotopie de $H$ est pseudoAnosov. En effet le théorème de classification de Thurston implique que si $H$ n'est pas pseudo-Anosov, alors l'action de $H$ sur $\mathcal{S}(M)$ admet une orbite périodique, mais dans ce cas les deux suites $\left(i\left(H^{n}(\gamma), \mathcal{H}\right)\right)_{n \in \mathbb{N}}$ et $\left.i\left(H^{n}(\gamma), \mathcal{V}\right)\right)_{n \in \mathbb{N}}$ ne prennent qu'un nombre fini de valeurs et par conséquent leur somme ne peut pas tendre vers l'infini.

\section{La représentation matricielle}

La démonstration du lemme suivant est immédiate.

Lemme 5.1. - Soit $x$ un chemin strictement positif. Si $1 \leq j \leq k$ et a est un segment contenu dans une feuille verticale dont aucune des extrémités n'est dans l'intérieur de l'anneau de feuilles horizontales homotopes à $c_{j}$ on $a$ :

$$
\#\left(T_{j} x \cap a\right)=\#(x \cap a)+\#\left(x \cap c_{j}\right) \#\left(c_{j} \cap a\right) .
$$

De même, si $1 \leq h \leq \ell$ et $b$ est un segment contenu dans une feuille horizontale dont aucune des extrémités n'est dans l'intérieur de l'anneau de feuilles verticales homotopes à $d_{h}$, on $a$ :

$$
\#\left(S_{h}^{-1} x \cap b\right)=\#(x \cap b)+\#\left(x \cap d_{h}\right) \#\left(d_{h} \cap b\right) .
$$

BULLETIN DE LA SOCIÉTÉ MATHÉMATIQUE DE FRANCE 
Remarquons que, dans le lemme précédent, $a$ (resp. $b$ ) peut être une feuille lisse fermée du feuilletage vertical (resp. horizontal).

Définissons les $(k+\ell, k+\ell)$-matrices $M\left(T_{j}\right)$ et $M\left(S_{h}^{-1}\right)$ par

$$
M\left(T_{j}\right)=\left(\begin{array}{cc}
\mathrm{Id}_{\mathrm{k}} & 0 \\
N\left(T_{j}\right) & \mathrm{Id}_{\ell}
\end{array}\right), \quad M\left(S_{h}^{-1}\right)=\left(\begin{array}{cc}
\operatorname{Id}_{\mathrm{k}} & N\left(S_{h}\right) \\
0 & \mathrm{Id}_{\ell}
\end{array}\right),
$$

où $\operatorname{Id}_{n}$ est la $n$-matrice carrée identité et

$$
\begin{gathered}
N\left(T_{j}\right)=\left(\begin{array}{ccccc}
0 & \cdots & i\left(\gamma_{j}, \delta_{1}\right) & \cdots & 0 \\
\vdots & & \vdots & & \vdots \\
0 & \cdots & i\left(\gamma_{j}, \delta_{\ell}\right) & \cdots & 0
\end{array}\right), \\
N\left(S_{h}\right)=\left(\begin{array}{ccccc}
0 & \cdots & i\left(\gamma_{1}, \delta_{h}\right) & \cdots & 0 \\
\vdots & & \vdots & & \vdots \\
0 & \cdots & i\left(\gamma_{k}, \delta_{h}\right) & \cdots & 0
\end{array}\right) .
\end{gathered}
$$

Supposons maintenant que $H$ est un mot en les $T_{j}$ et en les $S_{h}^{-1}$. On définit la matrice $M(H)$ comme le produit correspondant des $M\left(T_{j}\right)$ et $M\left(S_{h}^{-1}\right)$.

Par le Lemme 5.1, nous obtenons :

Lemme 5.2. - Si $\gamma$ est une classe d'isotopie de courbes, notons $I(\gamma)$ le vecteur de composantes $\left(i\left(\gamma_{1}, \gamma\right), \ldots, i\left(\gamma_{k}, \gamma\right), i\left(\delta_{1}, \gamma\right), \ldots, i\left(\delta_{\ell}, \gamma\right)\right)$. Pour tout mot $H$ en les $T_{j}$ et en les $S_{h}^{-1}$ et toute courbe fermée strictement positive $x$, on a $I(H([x]))=M(H) I([x])$, où $[x]$ est la classe d'isotopie de $x$.

Proposition 5.3. - Si $\mathrm{H}$ est un bon mot, toutes les grandes puissances de $M(H)$ sont strictement positives.

Preuve. - Puisque $H$ est un bon mot, il contient au moins une occurence de chaque $T_{j}$ et de chaque $S_{h}^{-1}$. L'inégalité

$$
\left(\operatorname{Id}_{k+\ell}+A\right)\left(\operatorname{Id}_{k+\ell}+B\right) \geq \operatorname{Id}_{k+\ell}+A+B,
$$

où $A$ et $B$ sont des matrices positives, montre que nous avons

$$
M(H) \geq\left(\begin{array}{cc}
\operatorname{Id}_{k} & \mathbf{I} \\
{ }^{t} \mathbf{I} & \operatorname{Id}_{\ell}
\end{array}\right)
$$

où $\operatorname{Id}_{n}$ est la $n$-matrice carrée identité et $\mathbf{I}$ est la matrice d'intersection

$$
\mathbf{I}=\left(\begin{array}{ccc}
i\left(\gamma_{1}, \delta_{1}\right) & \cdots & i\left(\gamma_{1}, \delta_{\ell}\right) \\
\vdots & \ddots & \vdots \\
i\left(\gamma_{k}, \delta_{1}\right) & \cdots & i\left(\gamma_{k}, \delta_{\ell}\right)
\end{array}\right)
$$

TOME $120-1992-\mathrm{N}^{\circ} 4$ 
Il suffit donc de montrer que la matrice

$$
J=\left(\begin{array}{cc}
\operatorname{Id}_{k} & \mathbf{I} \\
{ }^{t} \mathbf{I} & \mathrm{Id}_{\ell}
\end{array}\right)
$$

a toutes ses grandes puissances strictement positives.

Montrons, d'abord, qu'il y a un entier $n_{0} \in \mathbb{N}$ tel que, pour tout $n \geq n_{0}$, les matrices $\left({ }^{t} \mathbf{I I}\right)^{n}$ et $\left(\mathbf{I}^{t} \mathbf{I}\right)^{n}$ soient strictement positives.

En effet, puisque chaque $c_{j}$ intersecte au moins un $d_{h}$, les coefficients diagonaux de $\mathbf{I}^{t} \mathbf{I}$ sont strictement positifs. De plus, si l'anneau horizontal de feuilles parallèles à $c_{j}$ et l'anneau horizontal de feuilles parallèles à $c_{j^{\prime}}$ ont un segment en commun, alors l'élément $\left(j, j^{\prime}\right)$ de $\mathbf{I}^{\mathbf{t}} \mathbf{I}$ n'est pas nul, puisque $c_{j}$ et $c_{j^{\prime}}$ intersectent au moins un $d_{h}$ commun. Il est facile de conclure, par connexité de la surface $M$, que toutes les grandes puissances de ${ }^{t} \mathbf{I I}$ et de $\mathbf{I}^{t} \mathbf{I}$ sont strictement positives.

Puisque tous les termes diagonaux de $J$ sont 1 , il suffit de montrer qu'une des puissances de $J$ est strictement positive. Nous avons :

$$
J^{2}=\left(\begin{array}{cc}
\operatorname{Id}_{k}+\mathbf{I}^{t} \mathbf{I} & 2 \mathbf{I} \\
2{ }^{t} \mathbf{I} & \operatorname{Id}_{\ell}+{ }^{t} \mathbf{I} \mathbf{I}
\end{array}\right) \geq\left(\begin{array}{cc}
\mathbf{I}^{t} \mathbf{I} & 0 \\
0 & { }^{t} \mathbf{I I}
\end{array}\right)
$$

Ceci donne pour tout $k \in \mathbb{N}$ :

$$
J^{2 k+1} \geq\left(\begin{array}{cc}
\left(\mathbf{I}^{t} \mathbf{I}\right)^{k} & \left(\mathbf{I}^{t} \mathbf{I}\right)^{k} \mathbf{I} \\
\left({ }^{t} \mathbf{I} \mathbf{I}\right)^{k}{ }^{t} \mathbf{I} & \left({ }^{t} \mathbf{I} \mathbf{I}\right)^{k}
\end{array}\right)
$$

Puisque chaque ligne et chaque colonne de $\mathbf{I}$ contient des termes strictement positifs, on conclut que $J^{2 k+1}$ est strictement positive pour $2 k+1$ assez grand.

Il en résulte, voir [FLP, exposé 12], que l'on a :

ThÉorème 5.4. - Si H est un bon mot, le coefficient de dilatation du pseudo-Anosov dans la classe de $H$ est donné par la plus grande valeur propre de la matrice $M(H)$. 


\section{Le représentant $H$ est presque pseudo-Anosov}

En géneral, un bon mot $H$ n'est pas, à conjugaison topologique près, le représentant pseudo-Anosov dans sa classe d'isotopie, comme le montre l'exemple suivant :

Exemple 6.1. - Supposons que la courbe $c_{1}$ intersecte $d_{1}$ et une autre courbe $d_{\ell}$. Si on pose $n=\sum_{h} i\left(\gamma_{1}, \delta_{h}\right) \geq 2$, on peut trouver une isométrie de $[0,1] \times \mathbb{R} / n \mathbb{Z}$ sur la réunion des carrés, construits au $\S 1$, qui intersectent $c_{1}$ et telle que $d_{1}$ intersecte l'image de $[0,1] \times[0,1]$ mais n'intersecte pas l'image de $[0,1] \times[1,2]$. Cette isométrie nous donne donc des coordonnées dans l'anneau de feuilles horizontales lisses homotopes à $c_{1}$. Dans ces coordonnées, le twist $T_{1}$ est donné par la formule $(x, y) \mapsto(x, y+n x)$. Fixons un entier $p$ et définissons pour $q=0, \ldots, p-1, x_{q}=1 / p n+q / p$. Si on appelle $\alpha_{q}$ le segment horizontal image de $\left\{x_{q}\right\} \times[0,1]$, on a :

$$
T_{1}^{p(n-1)} S_{1}^{-1} T_{1}^{p} \alpha_{q}=\mathrm{id} .
$$

En effet, on a :

$$
T_{1}^{p}\left(x_{q}, y\right)=\left(x_{q}, y+p n x_{q}\right)=\left(x_{q}, y+1\right) .
$$

Or, si $0 \leq y \leq 1$, le point $\left(x_{q}, y+1\right)$ est dans la région où $S_{1}$ est l'identité; de plus :

$$
T_{1}^{p(n-1)}\left(x_{q}, y+1\right)=\left(x_{q}, y+1+p(n-1) n x_{q}\right)=\left(x_{q}, y\right) .
$$

Considérons alors le bon mot $H=S_{2}^{-1} \cdots S_{\ell}^{-1} T_{2} \cdots T_{k} T_{1}^{p(n-1)} S_{1}^{-1} T_{1}^{p}$. On voit facilement que sur chacun des segments $\alpha_{q}$, le mot $H$ est l'identité.

Montrons maintenant que le type de comportement de l'exemple précédent est la seule chose qui empêche un bon mot $H$ d'être pseudoAnosov.

Proposition 6.2. - Soit $H$ un bon mot. On peut trouver un nombre fini $\sigma_{i}^{v}$ de segments, deux à deux disjoints, contenus dans des feuilles lisses fermées verticales et un nombre fini $\sigma_{j}^{h}$ de segments deux à deux disjoints, contenus dans des feuilles lisses fermées horizontales qui verifient les propriétés suivantes :

(i) les segments horizontaux et les segments verticaux sont disjoints;

(ii) Si g est une géodésique de la structure plate, on a

$$
\sup _{n \in \mathbb{Z}} \ell_{s}\left(H^{n}(g)\right)<+\infty
$$

si et seulement si $g$ est contenu dans un $\sigma_{i}^{v}$ ou dans un $\sigma_{j}^{h}$.

TOME $120-1992-\mathrm{N}^{\circ} 4$ 
Preuve. - Par le même raisonnement que celui utilisé au $\S 4$ pour la démonstration du théorème, on déduit de 3.2 et de 3.4 que, si $\sup _{n \in \mathbb{Z}} \ell_{s}\left(H^{n}(g)\right)<+\infty$, alors la réunion des $H^{n}(g)$ est contenue soit dans la même feuille fermée lisse du feuilletage horizontal, soit dans la même feuille fermée lisse du feuilletage vertical. Si $c$ est une feuille fermée lisse du feuilletage, par exemple, horizontal, les composantes connexes non triviales de $\bigcap_{n \in \mathbb{Z}} H^{n}(c)$ forment une famille au plus dénombrable $\left(\sigma_{i}\right)_{i \in I}$ de segments, deux à deux disjoints, qui sont permutés par $H$. De plus, si $c$ est dans l'anneau de feuille homotope à $c_{p}$, l'effet de $H$ sur la réunion $\bigcup_{i \in I} \sigma_{i}$ est le même que l'effet de $T_{p}^{n_{H, p}}$, où $n_{H, p}$ est le nombre de fois où $T_{p}$ apparaît dans le mot $H$. Il en résulte que la restriction de $\mathrm{H}$ à $\bigcup_{i \in I} \sigma_{i}$ est la restriction de la rotation $T_{p}^{n_{H, p}}$ de $c$, la distance sur $c$ étant donnée par la mesure transverse du feuilletage vertical. Puisque, par 3.2, la réunion $\bigcup_{i \in I} \sigma_{i}$ n'est pas $c$ en entier, cette rotation est rationnelle. Remarquons maintenant que toute composante du complémentaire de la réunion $\bigcup_{i \in I} \sigma_{i}$ est nécessairement de longueur au moins 1, mesurée par la mesure transverse au feuilletage vertical. En effet, le support d'un $S_{\ell}$ intersecte $c$ en une réunion de segments chacun de longueur 1 . On en conclut que la famille $\sigma_{i}$ est finie, le nombre de segments étant borné $\operatorname{par} \sum_{\ell=1}^{\ell=h} i\left(\gamma_{p}, \delta_{\ell}\right)$.

Il est aisé de conclure de ce qui précède, que le phénomène décrit plus haut ne peut se passer que pour un nombre fini de feuilles du feuilletage horizontal (ou vertical).

Appelons $\left(\sigma_{i}^{h}\right)_{i \in I}$ la famille finie des segments maximaux horizontaux tels que $\forall n \in \mathbb{Z}, \ell_{s}\left(H^{n}\left(\sigma_{i}^{h}\right)\right)=\ell_{s}\left(\sigma_{i}^{h}\right)$. De même, appelons $\left(\sigma_{j}^{v}\right)_{j \in J}$ la famille finie des segments maximaux verticaux tels que

$$
\forall n \in \mathbb{Z}, \quad \ell_{s}\left(H^{n}\left(\sigma_{j}^{v}\right)\right)=\ell_{s}\left(\sigma_{j}^{v}\right) .
$$

Remarquons qu'un segment vertical $\sigma_{j}^{h}$ et un segment horizontal $\sigma_{i}^{h}$ ne peuvent pas s'intersecter. En effet, s'ils s'intersectaient en un point $m_{0}$, prenons un point $m_{1}$ près de $m_{0}$ sur le segment vertical et un point $m_{2}$ près de $m_{0}$ sur le segment horizontal. Dénotons par $\left[m_{i}, m_{k}\right]$ la géodésique de la structure plate entre $m_{i}$ et $m_{k}$. La géodésique $\left[m_{1}, m_{2}\right]$ est soit strictement positive soit strictement négative, par conséquent, $\sup _{n \in \mathbb{Z}} \ell_{s}\left(H^{n}\left(\left[m_{1}, m_{2}\right]\right)\right)=\infty$. Par ailleurs,

$$
\begin{aligned}
\ell_{s}\left(H^{n}\left(\left[m_{1}, m_{2}\right]\right)\right) & \leq \ell_{s}\left(H^{n}\left(\left[m_{1}, m_{0}\right]\right)\right)+\ell_{s}\left(H^{n}\left(\left[m_{0}, m_{2}\right]\right)\right) \\
& =\ell_{s}\left(\left[m_{1}, m_{0}\right]\right)+\ell_{s}\left(\left[m_{0}, m_{2}\right]\right) .
\end{aligned}
$$

Il est facile de construire une application $\theta: M \rightarrow M$ homotope à l'identité telle que les préimages de $\theta$ non réduites à un point soient les $\sigma_{i}^{h}$ 
et les $\sigma_{j}^{v}$. Puisque $H$ permute les $\sigma_{i}^{h}$ ainsi que les $\sigma_{j}^{v}$, on peut définir un homémorphisme $H_{0}$ de $M$ tel que $H_{0} \theta=\theta H$. Puisque $\theta$ est homotope à l'identité, l'homéomorphisme $H_{0}$ est homotope à $H$.

Nous allons montrer que $H_{0}$ est topologiquement conjugué au pseudoAnosov dans sa classe d'homotopie. Nous aurons besoin du lemme suivant :

Lemme 6.3. - Soit $f: M \rightarrow M$ un pseudo-Anosov. Soit $g: M \rightarrow M$, homotope à $f$, admettant un relevé $\tilde{g}$ au revêtement universel $\widetilde{M}$ de $M$, tel que

$$
\forall m_{1} \neq m_{2} \in \widetilde{M}, \quad \sup _{n \in \mathbb{Z}} \tilde{d}\left(\tilde{g}^{n}\left(m_{1}\right), \tilde{g}^{n}\left(m_{2}\right)\right)=\infty,
$$

où $\tilde{d}$ est une métrique sur $\widetilde{M}$ invariante par $\pi_{1}(M)$ le groupe $d u$ revêtement $\widetilde{M} \rightarrow M$. Alors $g$ est topologiquement conjugué à $f$ par une conjugaison homotope à l'identité.

Preuve. - Par [Ha] on peut trouver $A \subset M$ un compact invariant par $g$ et une application $\alpha: A \rightarrow M$ surjective homotope à l'inclusion $A \subset M$ qui semi-conjugue $g_{\mid A}$ à $f$. Montrons que $\alpha$ est, en fait, injective. En effet, appelons $p: \widetilde{M} \rightarrow M$ la projection du revêtement universel $\widetilde{M}$ sur $M$. Posons $\widetilde{A}=p^{-1}(A)$, si $\alpha_{t}$, avec $0 \leq t \leq 1$, est une homotopie de l'inclusion $A \subset M$ à $\alpha$, relevons-la en une homotopie $\widetilde{\alpha}_{t}$ telle que $\alpha_{0}$ soit l'inclusion $\widetilde{A} \subset \widetilde{M}$. Posons $\widetilde{\alpha}=\widetilde{\alpha}_{1}$. Puisque la métrique $d$ est invariante par les transformations du revêtement, la compacité de $A$ montre que l'on a :

$$
K=\sup _{x \in \widetilde{A}} d(x, \widetilde{\alpha}(x))<\infty .
$$

On peut trouver un relevé $\tilde{f}$ de $f$ à $\widetilde{M}$ tel que $\widetilde{\alpha} \tilde{g}=\tilde{f} \widetilde{\alpha}$. Si $x, y \in M$ sont tels que $\alpha(x)=\alpha(y)$, on peut trouver des relevés $\tilde{x}, \tilde{y} \in \widetilde{M}$ tels que $\widetilde{\alpha}(\tilde{x})=\widetilde{\alpha}(\tilde{y})$ (on utilise ici, que le choix de $\widetilde{\alpha}$ implique que $\widetilde{\alpha} T=T \widetilde{\alpha}$, pour tout automorphisme $T$ du revêtement $\widetilde{M} \rightarrow M$ ). On a alors :

$$
\forall n \in \mathbb{Z}, \quad \widetilde{\alpha} \tilde{g}^{n}(\tilde{x})=\tilde{f}^{n} \widetilde{\alpha}(\tilde{x})=\tilde{f}^{n} \widetilde{\alpha}(\tilde{y})=\widetilde{\alpha} \tilde{g}^{n}(\tilde{y}) .
$$

D'où pour tout $n \in \mathbb{Z}, d\left(g^{n}(\tilde{x}), g^{n}(\tilde{y})\right) \leq 2 K$. Par la propriété $\left(^{*}\right)$, on obtient $\tilde{x}=\tilde{y}$.

Puisque $\alpha: A \rightarrow M$ est injective et surjective, son domaine $A$ est homéomorphe à $M$. Comme $A \subset M$, le théorème de l'invariance du domaine montre que $M=A$.

Finissons de montrer que $H_{0}$ est topologiquement conjugué à un pseudo-Anosov. 
On peut, bien sûr relever la structure plate sur $M$, définie au $\S 1$, en une structure plate sur $\widetilde{M}$. Si $a, b$ sont dans $\widetilde{M}$, posons

$$
d(a, b)=\ell_{s}(\beta)
$$

où $\beta$ est la géodésique, pour la structure plate sur $\widetilde{M}$, joignant $a$ à $b$. Il est bien connu que $d$ est une distance définissant la topologie de $\widetilde{M}$; elle est, de plus, clairement invariante par les transformations du revêtement $\widetilde{M}$. Puisque $\theta$ est homotope à l'identité, par un argument déjà vu dans le lemme précédent, on peut trouver un relevé $\tilde{\theta}: \widetilde{M} \rightarrow \widetilde{M}$ tel que $K_{1}=\sup _{x \in \widetilde{M}} d(\theta(x), x)<\infty$. Par un autre argument du Lemme 6.3, on a des relevés $\widetilde{H}$ et $\widetilde{H}_{0}$ de $H$ et $H_{0}$, tels que $\widetilde{H}_{0} \tilde{\theta}=\tilde{\theta} \widetilde{H}$. Si $m_{1}, m_{2} \in \widetilde{M}$ sont tels que

$$
\sup _{n \in \mathbb{Z}} d\left(\widetilde{H}_{0}^{n}\left(m_{1}\right), \widetilde{H}_{0}^{n}\left(m_{2}\right)\right)<\infty
$$

choisissons $m_{1}^{\prime}$ et $m_{2}^{\prime}$ tels que $m_{1}=\tilde{\theta}\left(m_{1}^{\prime}\right)$ et $m_{2}=\tilde{\theta}\left(m_{2}^{\prime}\right)$ (ceci est possible parce que $\tilde{\theta}$ est proprement homotope à l'identité donc surjective). De l'égalité $\widetilde{H}_{0} \tilde{\theta}=\tilde{\theta} \widetilde{H}$ et $\sup _{x \in \widetilde{M}} d(\theta(x), x)<\infty$, il résulte que l'on a :

$$
\sup _{n \in \mathbb{Z}} d\left(\widetilde{H}^{n}\left(m_{1}^{\prime}\right), \widetilde{H}^{n}\left(m_{2}^{\prime}\right)\right)<\infty
$$

Si $g$ est la géodésique de la structure plate de $\widetilde{M}$, un argument similaire à la démonstration du théorème du $\S 4$, montre que l'on doit avoir $\sup _{n \in \mathbb{Z}} \ell_{s}\left(\widetilde{H}^{n}(g)\right)<\infty$. On conclut aisément de 6.1 , que $g$ doit être contenu dans un des relevés des $\sigma_{i}^{h}$ ou des $\sigma_{j}^{v}$; donc $\tilde{\theta}(g)$ est réduit à un point, par conséquent $m_{1}=m_{2}$.

On peut, alors, résumer la situation ainsi :

ThÉORÈme 6.4. - Soit $H$ est un bon mot et $A$ le pseudo-Anosov dans sa classe d'homotopie. Il existe une semi-conjugaison $\theta: M \rightarrow M$ homotope à l'identité telle que $A \theta=\theta H$. De plus, les préimages $\theta^{-1}(x), x \in M$ sont réduites à un point sauf, pour éventuellement un nombre fini de points de $M$, où elles sont homéomorphes à un intervalle. 


\section{Commentaires}

Le Lemme 6.3 est un argument qui est essentiellement dû à Lewowicz [Le1], [LS]. C'est aussi une conséquence du théorème de HirAide [Hi] et LEWOWICZ [Le2], qui établit que les homéomorphismes expansifs d'une surface compacte sont précisement les pseudo-Anosov. Nous avons préféré le déduire du travail plus court de HANDEL [Ha]. Le lecteur remarquera que l'on peut obtenir une démonstration du théorème de Penner, en établissant que $H_{0}$ est expansif et en faisant appel au théorème de HiraIDE et LEwowicz.

Dans le semi-groupe du groupe des homéomorphismes engendré par les $T_{j}$ et les $S_{h}$, on a un idéal bilatère d'homéomorphismes tous conjugués à des pseudo-Anosov. Cet idéal consiste en les composés $H$ tels que pour tout segment $\alpha$ horizontal ou vertical on a $\ell_{s}(H(\alpha))>0$. En particulier, le composé $T_{1} \cdots T_{k} S_{1}^{-1} \cdots S_{h}^{-1}$ est contenu dans cet idéal.

\section{BIBLIOGRAPHIE}

[Fa] Fathi (A.). - Dehn twists and pseudo-Anosov diffeomorphisms, Invent. Math., t. 87, 1987, p. 129-151.

[FlP] Fathi (A.), Laudebnbach (F.) et Poenaru (V.). - Travaux de Thurston sur les surfaces, Astérisque, t. 66-67, 1979.

[Ha] Handel (M.). - Global shadowing of pseudo-Anosov homeomorphisms, Ergodic Theory Dynamical Systems, t. 5, 1985, p. 373-377.

[Hi] Hiraide (K.). - Expansive homeomorphisms of compact surfaces are pseudo-Anosov, Osaka J. Math., t. 27, 199o, p. 117-162.

[Le1] Lewowicz (J.). - Persistence in Expansive Systems, Ergodic Theory Dynamical Systems, t. 3, 1983, p. 567-578.

[Le2] Lewowicz (J.). - Expansive homeomorphisms of surfaces, Bol. Soc. Brasil. Mat., t. 20, 1989, p. 113-133.

[LS] Lewowicz (J.) and Lima (E.). - Analytic models of pseudo-Anosov maps, Ergodic Theory Dynamical Systems, t. 6, 1986, p. 385-392.

[Pe] Penner (R.). - A construction of pseudo-Anosov homeomorphisms, Trans. Amer. Math. Soc., t. 310, 1988, p. 179-197.

[St] Strebel (K.). - Quadratic differentials. - Springer-Verlag, NewYork, Heidelberg, Berlin, Tokyo, 1984.

TOME $120-1992-\mathrm{N}^{\circ} 4$ 